\title{
Produção, caracterização e avaliação in vitro de partículas de quitosana e hidroxiapatita para substituição óssea
}

\section{(Development, characterization and in vitro evaluation of chitosan and hydroxyapatite particles for bone replacement)}

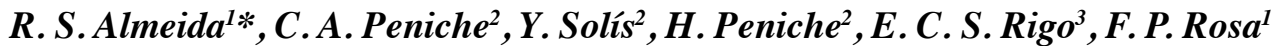 \\ ${ }^{I}$ Universidade Federal da Bahia, Instituto de Ciências da Saúde, Laboratório de Bioengenharia Tecidual e \\ Biomateriais, Av. Reitor Miguel Calmon, s/n, 40110-100, Salvador, BA, Brasil \\ ${ }^{2}$ Universidad de La Habana, Centro de Biomateriales, Havana, Cuba \\ ${ }^{3}$ Universidade de São Paulo, Faculdade de Zootecnia e Engenharia de Alimentos, Pirassununga, SP, Brasil
}

\begin{abstract}
Resumo
Objetivou-se desenvolver, caracterizar e avaliar in vitro biomateriais compósitos de quitosana com hidroxiapatita (CHI/HA) para atuarem como substitutos ósseos. As quitosanas de baixa (L-CHI) e média (M-CHI) massa molecular foram previamente caracterizadas por gravimetria, viscosimetria e espectrofotometria ultravioleta. Os compósitos de CHI/HA foram produzidos por precipitação in situ, seguida de centrifugação (micropartículas) ou spray drying (nanopartículas), e caracterizados por microscopia eletrônica de varredura, espectroscopia de infravermelho com transformada de Fourier e difração de raios X. A citotoxicidade in vitro foi determinada por teste de viabilidade celular. L-CHI e M-CHI apresentaram, respectivamente, 15,7\% e 16,4\% de umidade, $0,51 \%$ e $0,18 \%$ de cinzas, $79,5 \%$ e $77,7 \%$ de grau de desacetilação e 4,18.104 e 17,3.104 de massa molecular média viscosimétrica. Os compósitos exibiram grupos característicos dos materiais de origem e escassa citotoxicidade. Concluiu-se que as técnicas foram eficazes para produção dos biomateriais, com características físico-químicas e biológicas adequadas para utilização nas terapias ósseas.
\end{abstract}

Palavras-chave: biomateriais, compósitos, hidroxiapatita, quitosana, nanoestruturas.

\begin{abstract}
It was aimed to develop, characterize and evaluate in vitro the biomaterial composites of chitosan with hydroxyapatite (CHI/HA) to be applied as bone substitutes. Chitosans of low ( $\mathrm{L}-\mathrm{CHI})$ and medium (M-CHI) molecular weight were previously characterized by the gravimetry, viscosimetry and ultraviolet spectrophotometry. The composites of CHI/HA were produced by in situ precipitation, followed by centrifugation (microparticles) or spray drying (nanoparticles), and characterized by scanning electron microscopy, Fourier-transform infrared spectroscopy and X-ray diffraction. In vitro cytotoxicity was determined by the cell viability test. The L-CHI and M-CHI presented, respectively, $15.7 \%$ and $16.4 \%$ of humidity, $0.51 \%$ and $0.18 \%$ ash, $79.5 \%$ and $77.7 \%$ deacetylation degree, and $4.18 \times 10^{4}$ and $17.3 \times 10^{4}$ mean molecular weight by viscosimetry. The composites exhibited characteristic groups of the source materials and scarce cytotoxicity. It was concluded that the techniques were effective for the production of biomaterials, with adequate physicochemical and biological characteristics for use in bone therapies.
\end{abstract}

Keywords: biomaterials, composites, hydroxyapatite, chitosan, nanostructures.

\section{INTRODUÇÃO}

O desenvolvimento de novos biomateriais tem sido de vital importância para a melhoria da qualidade de vida de indivíduos acometidos por doenças degenerativas, lesões teciduais, dentre outros. Estes biomateriais podem ser qualquer substância, natural ou sintética, que possa ser utilizada de forma temporária ou definitiva, como parte de um sistema que objetiva restaurar ou substituir qualquer tecido, órgão ou função do corpo [1]. Em meio a diversas classificações, os biomateriais podem ser agrupados

*renata.almeidabio@gmail.com

Dhttps://orcid.org/0000-0001-8359-6345 como metálicos, cerâmicos, poliméricos ou compósitos (associação de dois ou mais tipos diferentes de materiais). A bioengenharia tecidual visa desenvolver novos biomateriais com alta bioatividade, de modo a promover a adesão e a migração celular, e fornecer fatores bioquímicos para a difusão de nutrientes necessários à célula, além de ter funções mecânicas, e estes processos podem ser avaliados in vitro e in vivo [2]. Um dos biomateriais mais utilizados nas aplicações clínicas para substituição óssea e dentária é a hidroxiapatita $\left[\mathrm{HA}, \mathrm{Ca}_{10}\left(\mathrm{PO}_{4}\right)_{6}(\mathrm{OH})_{2}\right]$, por demonstrar forte biocompatibilidade com células e tecidos, ter capacidade de associação com moléculas, íons e metais, estabilidade no meio biológico, atividade osteocondutora e ser versátil no processamento em diferentes formatos (pó, blocos densos 
ou porosos, partículas) [3]. Entretanto, a HA possui algumas limitações que podem comprometer sua atividade biológica em algumas aplicações, como fragilidade mecânica, alta cristalinidade, baixa taxa de dissolução in vivo e migração das partículas do sítio de implante, o que pode causar danos ao tecido saudável [4]. Para resolver esta problemática, a HA pode ser incrementada ao ser associada a polímeros naturais ou sintéticos (colágeno, alginato, gelatina e quitosana).

Um polímero natural que têm desempenhado um papel significativo em muitas aplicações de engenharia de tecidos é a quitosana [CHI, $\left.\left(\mathrm{C}_{6} \mathrm{H}_{11} \mathrm{O}_{4} \mathrm{~N}\right)_{\mathrm{n}}\right]$, por ser biodegradável e atóxico. Trata-se de um polissacarídeo amino, obtido por $\mathrm{N}$-desacetilação em uma extensão variável da quitina, e tem a estrutura química primária formada pela repetição de unidades unidas por ligações $\beta(1 \rightarrow 4)$ 2-acetamida2 -deoxi- $\beta$-D-glucano (N-acetil-D-glucosamina) e $(1 \rightarrow 4)$ 2 -amino-2-deoxi- $\beta$-D-glucano (D-glucosamina) $[5,6]$. A quitina constitui a maior fração dos exoesqueletos de insetos e crustáceos, sendo considerada como o segundo polissacarídeo mais abundante da natureza, ficando atrás apenas da celulose [6]. Difere da CHI em relação à proporção de grupos acetamida e amino no polímero, o que resulta em diferentes solubilidades, ou seja, a quitina possui mais de $50 \%$ de grupos acetamida (N-acetil D-glucosamina) comparando-se aos grupos amino e é inerte e insolúvel na maioria dos solventes comuns. A CHI possui maior porcentagem de grupos amino (D-glucosamina) em relação aos grupos acetil, o que possibilita que ela seja reativa e solúvel em soluções aquosas de ácidos orgânicos e inorgânicos diluídos [7]. A composição das cadeias da $\mathrm{CHI}$ e suas dimensões geralmente variam dependendo do material de origem e do rigor do método de produção, de modo que o grau de desacetilação (DD) e a massa molecular $\left(\mathrm{M}_{\mathrm{v}}\right)$ são dois parâmetros de conhecimento obrigatório para caracterizar uma amostra desse polissacarídeo. Outras características como polidispersidade de sua massa molecular, teor de umidade, solubilidade e porcentagens de cinzas também são conhecimentos obrigatórios para muitas aplicações [8]. O uso de análises químicas, físicas e ópticas é importante para determinar as características da matériaprima e se estas foram mantidas durante o processamento do biomaterial.

Os compósitos de hidroxiapatita com quitosana apresentam características interessantes para a reparação do tecido ósseo, tendo em vista que sua composição química é similar ao osso, por isso são frequentemente utilizados como materiais de preenchimento na ortopedia. A quitosana apresenta biodegradabilidade, mucoadesividade, flexibilidade e restringe a migração das partículas para os tecidos circunjacentes, enquanto a hidroxiapatita proporciona estrutura, bioatividade e osteocondutividade [9]. Desta forma as propriedades funcionais dos materiais monofásicos podem ser aproveitadas em conjunto. Portanto, o desafio da pesquisa atual reside em produzir compósitos de biocerâmicas associadas a polímeros com alta taxa de degradação e com características ideais para estimular a neoformação tecidual, propiciar a formação de estruturas semelhantes aos elementos de matriz extracelular, facilitar a mobilização, expansão e integração de populações de células regenerativas e fomentar o reparo de lesões. Estas características estão intimamente relacionadas com o desempenho biológico, como bioatividade, condução e capacidade de dissolução após a implantação in vivo. Além disso, testes qualitativos de citotoxicidade in vitro (observação de mudanças morfológicas das células) e/ ou quantitativa (investigação de parâmetros celulares, como morte celular, inibição do crescimento, proliferação celular ou formação de colônias) são necessários para materiais com potencial para aplicações biomédicas. Inúmeras metodologias permitem produzir compósitos de $\mathrm{CHI}$ com HA, que variam desde misturas, revestimento, coprecipitação e biomineralização. Recentemente, uma nova rota foi adaptada para a produção destes compósitos. Tratase de uma abordagem biomimética, que permite controlar a arquitetura e a composição química dos biomateriais [9]. $\mathrm{O}$ método consiste na coprecipitação de fosfato dicálcico di-hidratado (DCPD) em solução ácida de CHI, juntamente com aumento gradual do $\mathrm{pH}$ do meio de reação pela adição de solução de hidróxido de sódio $(\mathrm{NaOH})[10,11]$. Esta técnica de biomimetismo in situ também pode simular a temperatura, força iônica e $\mathrm{pH}$ dos meios biológicos, o que faz com que os materiais produzidos possuam propriedades semelhantes ao tecido que se deseja recuperar. Outra técnica também adaptada à bioengenharia tecidual para coating de partículas com tamanhos controlados é o spray drying, que é amplamente utilizado na indústria alimentar e farmacêutica por ser de baixo custo, rápida execução e de fácil ampliação. Esta recente técnica utiliza um bico atomizador para gerar milhões de gotículas com elevada área superficial, que são rapidamente secas por um fluxo contracorrente de gás, com temperatura variando de 0 a $120^{\circ} \mathrm{C}$, o que permite a obtenção de um produto em escala micrométrica e nanométrica [12].

Existe grande empenho para aprimorar as propriedades físico-químicas dos biomateriais, como porosidade, densidade, cristalinidade, formato e tamanho das partículas, para que possam atuar como um mecanismo que envia sinais para os tecidos circunvizinhos e recrute células [13]. A área de superfície disponível para reagir com células e fluidos biológicos tem relação com o formato e tamanho do biomaterial; assim, partículas de tamanhos variados ao serem implantadas favorecem a formação de um interstício, uma vez que as partículas menores ocupam os intervalos entre as partículas maiores, o que possibilita a difusão de micronutrientes e fatores de crescimento, migração, proliferação, diferenciação celular e angiogênese [14]. Os biomateriais nanoestruturados possuem organização estrutural com partículas de tamanhos menores que $100 \mathrm{~nm}$ e apresentam propriedades diferentes de materiais em escalas maiores e de partículas agregadas. A HA nanoestruturada desempenha papel significativo nas terapias ósseas, devido às suas propriedades funcionais de alta relação superfície/ volume e estrutura semelhante a apatita biológica. Esta semelhança é crucial para as interações células-biomaterial e consequente regeneração [15]. Apesar dos avanços 
científicos, ainda são necessários constantes estudos para desenvolvimento de novos biomateriais, com propriedades similares ao tecido lesionado, em vista que ainda não existe um biomaterial que atenda todas as características físico-químicas ideais para aperfeiçoar a regeneração, principalmente de defeitos críticos. Neste contexto, o presente estudo teve como objetivo desenvolver, caracterizar as propriedades físico-químicas e avaliar a citotoxicidade in vitro de biomateriais compósitos de CHI, com baixa e média massa molecular, associada à HA nanoestruturada.

\section{MATERIAL E MÉTODOS}

Purificação da quitosana: utilizaram-se duas amostras de CHIs comerciais $\left[\left(\mathrm{C}_{6} \mathrm{H}_{11} \mathrm{O}_{4} \mathrm{~N}\right)_{\mathrm{n}}\right.$, Aldrich, Reino Unido], que segundo o fabricante possuem baixa (L-CHI, 5-19x10 $\mathrm{g} / \mathrm{mol}$ ) e média (M-CHI, $19-31 \times 10^{4} \mathrm{~g} / \mathrm{mol}$ ) massa molecular, e grau de desacetilação de 75-85\%. A CHI foi dissolvida em $2 \%(\mathrm{~m} / \mathrm{v})$ de ácido acético glacial aquoso $\left(\mathrm{CH}_{3} \mathrm{COOH}\right.$, Panreac, Espanha) até obtenção de uma solução homogênea a $0,5 \%(\mathrm{~m} / \mathrm{v})$, depois foi sucessivamente filtrada através de filtros de vidro (diâmetros dos poros: $45 \mathrm{a} 5 \mu \mathrm{m}$ ) e membranas porosas $(0,8$ a $0,45 \mu \mathrm{m})$. Deixou-se a solução precipitar por gotejamento em hidróxido de sódio $1 \mathrm{M}(\mathrm{NaOH}$, Sigma Chemical, Alemanha) até $\mathrm{pH} \sim 8$ e filtrou-se novamente. O sólido foi cuidadosamente lavado com água bidestilada até que nenhuma alteração na condutividade foi detectada, seguida de sucessivas lavagens com concentrações de $70 \%$, $80 \%, 90 \%$ e $100 \%$ de etanol (v/v, $\mathrm{C}_{2} \mathrm{H}_{6} \mathrm{O}$, Sigma Chemical, Alemanha) [9]. Por fim, o polímero purificado foi seco em vácuo a $25-28^{\circ} \mathrm{C}$.

Preparação dos compósitos: os compósitos foram preparados baseando-se num método descrito na literatura com algumas modificações [9-11], e consistiu em uma abordagem biomimética em dois estágios fundamentais: precipitação e hidrólise in situ da fase inorgânica (HA) dentro da matriz polimérica (CHI). A princípio prepararamse as soluções precursoras de cálcio [acetato de cálcio $5 \% \mathrm{~m} / \mathrm{v}, \mathrm{Ca}\left(\mathrm{CH}_{3} \mathrm{COO}\right)_{2}, \mathrm{BDH}$, Reino Unido] e de fósforo (di-hidrogenofosfato de sódio $5 \% \mathrm{~m} / \mathrm{v}, \mathrm{NaH}_{2} \mathrm{PO}_{4}$, Sigma Chemical, Alemanha). Em seguida empregaram-se duas técnicas para a preparação dos compósitos: técnica 1 centrifugação: preparou-se uma solução de CHI a $3 \%(\mathrm{~m} / \mathrm{v})$ em $1 \%$ de ácido acético glacial $(\mathrm{m} / \mathrm{v})$ e adicionaram-se $5 \%(\mathrm{~m} / \mathrm{v})$ do sal precursor de fósforo (di-hidrogenofosfato de sódio). Após homogeneização adicionou-se a solução precursora de cálcio e manteve-se sob agitação por $24 \mathrm{~h}$ para assegurar uma dispersão homogênea do DCPD (fase precursora da HA) precipitado na solução viscosa de CHI (suspensão CHI/DCPD). Para a produção das partículas com concentração de $20 \%$ de CHI e $80 \%$ de HA (L-CHI20/HA80 e M-CHI20/HA80), a solução de CHI/DCPD foi centrifugada e o sobrenadante descartado para obtenção do precipitado. Após hidrólise e secagem este precipitado foi macerado com pistilo até obtenção de um material homogêneo; técnica 2 spray drying: preparou-se uma solução de CHI a $2 \%(\mathrm{~m} / \mathrm{v})$ em $1 \%$ de ácido acético glacial $(\mathrm{m} / \mathrm{v})$, e adicionaram-se as soluções precursoras de cálcio e de fósforo, mantendo sob agitação constante para precipitação do DCPD na solução de CHI (suspensão CHI/DCPD). Para a produção das partículas com concentração de 50\% de CHI e 50\% de HA (L-CHI50/ HA50 e M-CHI50/HA50), a solução de CHI/DCPD foi pulverizada em um Buchi Mini Spray Dryer B-191 (Flawil, Suíça) com um bico padrão de $0,7 \mathrm{~mm}$. As condições de secagem do pulverizador foram definidas da seguinte forma: taxa de fluxo $32 \mathrm{~m}^{3} / \mathrm{h}$, taxa de fluxo de ar comprimido 473 $\mathrm{NL} / \mathrm{h}$ e temperatura de entrada $160^{\circ} \mathrm{C}$. Após produção, ambas as amostras foram submetidas ao processo de hidrólise, para obtenção da HA a partir do DCPD precipitado na matriz de CHI. Os materiais foram imersos em uma solução saturada de fosfato de sódio 8,8\% (m/v) e pH $12\left(\mathrm{Na}_{3} \mathrm{PO}_{4}\right.$, Riedel-de Haën, Alemanha), com solução de tripolifosfato de sódio a $1 \%\left(\mathrm{~m} / \mathrm{v}, \mathrm{Na}_{5} \mathrm{P}_{3} \mathrm{O}_{10}\right.$, Acros Organics, Bélgica), e mantidos em banho-maria a $60{ }^{\circ} \mathrm{C}$ por $72 \mathrm{~h}$. Por fim, as amostras foram filtradas e passaram por banhos de água fria $\left(\sim 10^{\circ} \mathrm{C}\right)$ e quente $\left(\sim 60^{\circ} \mathrm{C}\right)$ até a neutralidade do $\mathrm{pH}$ e secos em estufa a $50-60{ }^{\circ} \mathrm{C}$. As amostras produzidas passaram por uma malha granulométrica de $425-250 \mu \mathrm{m}$, depois foram fracionadas em eppendorfs e esterilizadas por radiação $\gamma$ com dose de $25 \mathrm{kGy}$.

Caracterização físico-química das quitosanas: determinou-se o teor de umidade $(\% \mathrm{H})$ por gravimetria, onde uma amostra de CHI previamente pesada foi seca em uma estufa (MLW WS100, Coimsa, México) a $105^{\circ} \mathrm{C}$ até atingir uma massa constante. $\mathrm{O} \% \mathrm{H}$ foi calculado a partir da diferença entre as massas da amostra úmida original $\left(\mathrm{W}_{\mathrm{i}}\right)$ e a amostra seca $\left(\mathrm{W}_{\mathrm{f}}\right)$, conforme:

$$
\% \mathrm{H}=\frac{\mathrm{w}_{\mathrm{i}}-\mathrm{w}_{\mathrm{f}}}{\mathrm{w}_{\mathrm{i}}} .100=\frac{\mathrm{w}_{\mathrm{H} 2 \mathrm{O}}}{\mathrm{w}_{\mathrm{i}}} .100
$$

onde $\mathrm{W}_{\mathrm{H} 2 \mathrm{O}}$ é o conteúdo de água. Também por gravimetria, obteve-se o percentual de cinzas $(\% \mathrm{Cz})$ ao colocar uma amostra de CHI previamente pesada em cadinhos tarados e levar a um forno tipo mufla H1064 (Termiber, Espanha) préaquecido a $900{ }^{\circ} \mathrm{C}$ durante $6 \mathrm{~h}$. Os cadinhos foram deixados no forno para resfriar até $200{ }^{\circ} \mathrm{C}$ e depois colocados em dessecadores com um topo ventilado. $\mathrm{O}$ teor de cinzas foi calculado conforme:

$$
\% \mathrm{Cz}=\frac{\mathrm{w}_{\mathrm{cz}}}{\mathrm{w}_{\mathrm{in}}} .100
$$

onde $\mathrm{W}_{\mathrm{Cz}}$ é a massa das cinzas e $\mathrm{W}_{\text {in }}$ é a massa inicial. A média da massa molecular viscosimétrica $\left(\mathrm{M}_{\mathrm{v}}\right)$ foi calculada a partir da viscosidade intrínseca [ $\eta$ ] de soluções de CHI em ácido acético $(0,3 \mathrm{M})$ e acetato de sódio $(0,2 \mathrm{M})$ a 25 ${ }^{\circ} \mathrm{C}$. As determinações foram realizadas em triplicata usando um viscosímetro de tipo Ubbelohde e $\mathrm{M}_{\mathrm{v}}$ foi calculada utilizando a equação Mark-Houwink-Sakurada [16]:

$$
[\eta]=K \cdot M_{v}{ }^{a}
$$

para um sistema CHI (solvente de $\mathrm{K}=0,076 \mathrm{~mL} / \mathrm{g}$ e $\mathrm{a}=0,76$ ). $\mathrm{O}$ grau de desacetilação (DD) foi determinado pelo método de primeira derivada por ultravioleta (UV) [17], que é 
uma medida quantitativa do número de grupos funcionais de amina no polímero. O DD foi calculado a partir de gravações da primeira derivada dos espectros UV de $\mathrm{N}$-acetil glucosamina das amostras de $\mathrm{CHI}$ a $202 \mathrm{~nm}$, usando cuvetes de quartzo ultravioleta com comprimento de $1 \mathrm{~cm}$, espectrofotômetro visível a UV Cintra 10e (GBC, Austrália) e o software UV-Spectrum Scan (GBC, Austrália).

Caracterização físico-química dos compósitos: a imagem macroscópica dos compósitos foi registrada por uma câmera digital D3200 (Nikkon, Japão) e a morfologia superficial foi analisada por microscopia eletrônica de varredura (MEV) com microscópio ambiental EVO LS 10 (Carl Zeiss, Alemanha). As amostras secas sem qualquer tratamento prévio foram montadas em um stub de alumínio e fixadas com fita adesiva de carbono e a observação foi realizada em condições de alto vácuo, utilizando um detector de elétrons secundários. A tensão de aceleração (EHT), o tamanho do ponto e as ampliações utilizadas estão especificadas nas micrografias. A análise por espectroscopia de infravermelho com transformada de Fourier (FTIR) foi obtida através da mistura dos compósitos com brometo de potássio (KBr, Sigma Chemical, Alemanha) para formação de discos e os espectros registrados na faixa de 4000 a 400 $\mathrm{cm}^{-1}$ utilizando o espectrômetro Tensor 27 FTIR (Bruker, Alemanha) e o software OPUS Data Collection Program (Bruker, Alemanha). Os espectros foram obtidos com uma resolução de $2 \mathrm{~cm}^{-1}$ e calculados com média de mais de 100 varreduras. O espectro da HA foi determinado a partir de uma amostra produzida anteriormente pelo grupo de pesquisa [9]. Os padrões de difração de raios X (DRX) dos diferentes biomateriais foram obtidos com um difratômetro D5005 (Siemens, Alemanha), com radiação $\mathrm{CuK} \alpha(\lambda=1,5406 \AA)$, tensão de $40 \mathrm{kV}$ e corrente de $15 \mathrm{~mA}$. A varredura angular

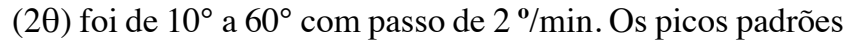
encontrados foram comparados aos disponíveis nos arquivos da JCPDS (n ${ }^{\circ} 46-0905$ para HA e $n^{\circ}$ 39-1894 para CHI). Para o cálculo do índice de cristalinidade (IC) das amostras, utilizaram-se os padrões de DRX, conforme a Eq. D [18], onde $\beta_{002}$ representa a largura à meia altura da reflexão do plano (002):

$$
\mathrm{IC}=\left(0.24 / \beta_{002}\right)^{3}
$$

Ensaios in vitro: o ensaio baseou-se na determinação de células viáveis após a exposição da população de células de ovário de hamster chinês (CHO-k1) a diversas concentrações do extrato (amostra dos biomateriais) [19]. Realizouse um monocultivo de CHO-k1 em placa de $150 \mathrm{~mm}$ de diâmetro com meio de cultura RPMI 1640 (Gibco, EUA), suplementado com penicilina $(100 \mathrm{U} / \mathrm{mL})$, estreptomicina $(100 \mu \mathrm{g} / \mathrm{mL})$, anfotericina B $(0,025 \mu \mathrm{g} / \mathrm{mL})$, glutamina $(2$ $\mathrm{mM}$ ) e soro fetal bovino (10\%), a $37^{\circ} \mathrm{C} \mathrm{em}$ atmosfera de $\mathrm{CO}_{2}$ (5\%) por $72 \mathrm{~h}$ em estufa incubadora CCL-170B-8 (ESCO, Dinamarca).Para garantir um crescimento celular satisfatório o meio de cultura suplementado (MCS) foi trocado nos períodos de 24 e $48 \mathrm{~h}$ e após $72 \mathrm{~h}$ o meio foi retirado e as células removidas com solução de tripsina Tryple Express
(Gibco, EUA) com vermelho de fenol 12605 (Gibco, EUA). A contagem celular foi realizada em câmara de Neubauer por diluição em azul de trypan 0,4\% (1:5) utilizando um microscópio óptico 8060A (Bel, Itália). O método é baseado na observação de células viáveis, impermeáveis ao corante, enquanto as células não viáveis permitem a penetração do corante devido à formação de poros na membrana, exibindo a coloração azul [20]. Para avaliação da viabilidade celular os materiais foram preparados conforme norma ISO $10993-$ 12 [21] e previamente esterilizados por radiação gama, com uma dose de $25 \mathrm{kGy}$. Todas as amostras foram adicionadas em criotubos e misturadas ao MCS na proporção $2 \%(\mathrm{~m} / \mathrm{v})$, em seguida incubadas em estufa a $37^{\circ} \mathrm{C}$ em atmosfera de $\mathrm{CO}_{2}(5 \%)$ por $24 \mathrm{~h}$. Após esse período as amostras foram homogeneizadas e centrifugadas a $13000 \mathrm{rpm}$ por $5 \mathrm{~min}$ em uma microcentrífuga CT 14000 (Cientec, Brasil) e o sobrenadante reservado para o ensaio. Utilizaram-se dois controles: 1) alumina (controle negativo); e 2) solução de fenol $0,2 \%$ (controle positivo). Após as $24 \mathrm{~h}$, tanto as amostras como os controles foram submetidos a diluições seriadas, obtendo-se as concentrações 100\%, 50\%, 25\%, 12,5\% e $6,25 \%$. O ensaio de citotoxicidade utilizado foi o método indireto de incorporação do vermelho neutro conforme norma ISO 10993-5 [22], onde células $\left(1.10^{4}\right.$ células/poço) foram cultivadas em MCS $(200 \mu \mathrm{L})$ e incubadas em estufa a $37{ }^{\circ} \mathrm{C}$ em atmosfera de $\mathrm{CO}_{2}(5 \%)$ por $24 \mathrm{~h}$ utilizando placa de 96 poços. Após esse período, o MCS foi retirado, os tratamentos (amostras e controles) e suas respectivas diluições $(200 \mu \mathrm{L})$ foram adicionados na placa contendo as células e foram novamente incubadas em estufa a 37 ${ }^{\circ} \mathrm{C}$ em atmosfera de $\mathrm{CO}_{2}(5 \%)$ por $24 \mathrm{~h}$. Após o tratamento observou-se a morfologia celular com microscópio óptico invertido Primo (Carl Zeiss, Alemanha) e o meio contendo os tratamentos foi retirado dos poços. Em seguida, as células foram lavadas com tampão fosfato salino (PBS, $200 \mu \mathrm{L}$ ). Após a retirada do PBS, adicionaram-se $200 \mu \mathrm{L}$ da solução de vermelho neutro $(50 \mu \mathrm{g} / \mathrm{mL})$ e para incorporação a placa foi incubada em estufa a $37{ }^{\circ} \mathrm{C}$ em atmosfera de $\mathrm{CO}_{2}$ (5\%) por $3 \mathrm{~h}$. A solução de vermelho neutro foi retirada e os poços lavados com $200 \mu \mathrm{L}$ de PBS, depois foram adicionados $200 \mu \mathrm{L}$ de solução de extração (50\% etanol:1\% ácido acético:49\% água destilada) e a placa submetida à agitação a $150 \mathrm{rpm}$ por $10 \mathrm{~min}$ em uma incubadora com agitação Shaker TE-420 (Tecnal, Brasil) para extração do vermelho neutro das células. As leituras das absorbâncias foram realizadas em triplicata para cada amostra, em leitor de microplacas Multiskan FC (Thermo Scientific, EUA) e os resultados foram expressos em viabilidade celular $(\% \mathrm{Vc})$, onde Abs amostra é a absorbância da amostra e Abs branco é a absorbância do branco, ambas a $540 \mathrm{~nm}$, conforme:

$$
\% \mathrm{Vc}=\frac{\text { Abs amostra }}{\text { Abs branco }} .100
$$

\section{RESULTADOS E DISCUSSÃO}

Caracterização das quitosanas: na Tabela I constam os resultados da avaliação físico-química das CHIs. Para 
determinar as características deste polímero e correlacionálas com as propriedades que podem resultar em aplicações biológicas, foi necessária uma prévia purificação, em vista que a utilização da $\mathrm{CHI}$ como biomaterial exige um grau extremamente alto de pureza e pouca quantidade de matéria inorgânica (menos de 1\%) [23]. Após criterioso processo de purificação, foram obtidas amostras uniformes e completamente solúveis de $\mathrm{CHI}$, com um rendimento (\%R) de $80,2 \%$ para L-CHI e $86,7 \%$ para $\mathrm{M}$-CHI, onde as perdas foram relacionadas aos materiais insolúveis e agregados excluídos após filtrações e a própria manipulação durante o experimento. A CHI de baixa massa molecular (L-CHI) teve menor rendimento devido a uma maior fluidez, mas os valores encontrados foram semelhantes ao reportado na literatura, quando foi comparada a eficácia de diferentes métodos de purificação [23]. Por gravimetria, os valores obtidos do teor de umidade $(\% \mathrm{H})$ foram semelhantes entre as duas amostras. Este valor é explicado pelo fato de que os grupos amino da $\mathrm{CHI}$ apresentam forte afinidade pela água e no estado sólido as macromoléculas podem apresentar mudanças na conformação, que tendem a adsorver água, sendo facilmente hidratadas [24]. Por ser um polímero natural, a CHI pode ter origem e composição variada e sua pureza depende do processo que foi realizado. O percentual de cinzas $(\% \mathrm{Cz})$ refere-se ao teor residual inorgânico que permanece após a queima da matéria orgânica e os valores encontrados foram baixos, obedecendo aos padrões aceitáveis para amostras ultrapuras $(<0.6 \%)$ e dentro dos valores para amostras comerciais $(<1 \%)$ [25].

A média da massa molecular viscosimétrica $\left(\mathrm{M}_{\mathrm{v}}\right)$ obtida confirmou que as CHIs possuíram baixa e média massa

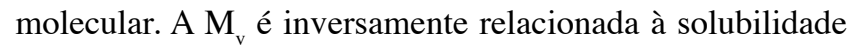
da $\mathrm{CHI}$, pois quanto maior a cadeia maior é sua $\mathrm{M}_{\mathrm{v}}$, o que favorece interações entre os segmentos e, consequentemente, diminui a solubilidade do polímero; desta forma pode-se concluir que a M-CHI pode ser menos solúvel em meios ácidos [26]. Outra característica avaliada foi o grau de desacetilação (DD), que é considerado um dos principais parâmetros na caracterização da CHI, sendo definido como o percentual de unidades do polímero que estão desacetiladas [27]. O DD também está relacionado com a solubilidade da CHI em meios ácidos, pois seu valor expressa a quantidade de grupos amino disponíveis para protonação e que geram cargas $\left(\mathrm{NH}_{3}^{+}\right)$na cadeia polimérica, em vista que, quanto maior o valor do DD, maior é a quantidade de cargas presentes na cadeia e ocorre mais repulsão eletrostática entre elas, consequentemente, maior é a solubilidade da CHI [26]. Desta forma, os DDs obtidos neste estudo foram inferiores aos observados por outros autores [26], o que sugere que as $\mathrm{CHI}$ utilizadas podem apresentar menor solubilidade em soluções de acidez moderada, e similares ao reportado em um estudo que avaliou CHIs comerciais de diferentes origens [28].

Caracterização dos compósitos: compósitos de $\mathrm{CHI} / \mathrm{HA}$ podem ser produzidos por diferentes técnicas, sendo que o método biomimético utilizado consistiu na precipitação in situ e a nucleação do componente inorgânico dentro da matriz polimérica. Este sistema é considerado mais vantajoso por permitir um melhor controle da microestrutura, homogeneidade e composição química dos materiais finais [10]. Os biomateriais produzidos possuíram um aspecto macroscópico de pó (Fig. 1), sendo que os produzidos pela técnica de spray drying, L-CHI50/HA50 (Fig. 1c) e M-CHI50/HA50 (Fig. 1d), aparentaram ser mais higroscópico, aglomerando-se com facilidade. A análise por MEV demonstrou que as partículas individuais dos compósitos produzidos se agregaram formando estruturas maiores, com tamanhos variados. Não foi observada segregação de cristais de HA, o que sugeriu que estavam depositados na matriz da CHI e juntos constituíram as partículas individuais. Notou-se que os compósitos produzidos pela técnica de centrifugação (Figs. 2a e 2b) possuíram tamanhos de partícula maiores (micropartículas de $\sim 5 \mu \mathrm{m}$ ) que os compósitos produzidos por spray drying (nanopartículas de $\sim 50 \mathrm{~nm}$ ), o que confirmou que o spray drying é viável para produção de materiais em escala nanométrica (Figs. 2c e 2d). Estes resultados demonstraram que ambas as técnicas proporcionaram a obtenção de partículas menores que em estudos anteriores ( 140 a $300 \mu \mathrm{m})$ $[9,11]$ em que foi empregado o método biomimético de precipitação in situ. Estudos referem que nanobiomateriais com partículas menores que $100 \mathrm{~nm}$ mimetizam melhor a microestrutura do tecido o que potencializa a interação celular [3, 29]; embora os potenciais benefícios dos materiais nanoestruturados e das nanopartículas sejam bem descritos, o impacto no organismo como um todo ainda não foi bem esclarecido. Em geral, o efeito das nanopartículas no corpo depende de propriedades como tamanho, massa, composição química, superfície e também como elas agem em conjunto, visto que, à medida que entram no organismo, os locais de penetração, a possibilidade de acumulação e a translocação destas nanopartículas podem determinar os riscos potenciais e toxicidade dos materiais [29]. Deste modo, são imprescindíveis estudos aprofundados a fim de tentar elucidar como os biomateriais nanométricos se comportam ao entrar em contato com meios biológicos e qual o tamanho de partícula ideal.

A espectroscopia na região do infravermelho permite

Tabela I - Resultados da caracterização físico-química das quitosanas de baixa (L-CHI) e média (M-CHI) massa molecular. [Table I - Results of physico-chemical characterization of chitosans of low (L-CHI) and medium (M-CHI) molecular weight.]

\begin{tabular}{cccccc}
\hline Amostra & $\% \mathrm{R}$ & $\% \mathrm{H}$ & $\% \mathrm{Cz}$ & $\mathrm{M}_{\mathrm{v}}$ & $\mathrm{DD}$ \\
\hline L-CHI & $80.2 \pm 0.3$ & $15.7 \pm 0.2$ & $0.51 \pm 0.01$ & $4.18 .10^{4}$ & $79.51 \pm 0.04$ \\
M-CHI & $86.7 \pm 2.7$ & $16.4 \pm 0.3$ & $0.18 \pm 0.04$ & $17.3 .10^{4}$ & $77.7 \pm 0.3$ \\
\hline
\end{tabular}

$\% R$ - percentual de rendimento ; $\% H$ - teor de umidade; $\% C z$ - percentual de cinzas; $M_{v}$ - média da massa molecular viscosimétrica; $D D$ - grau de desacetilação; $n=3$. 

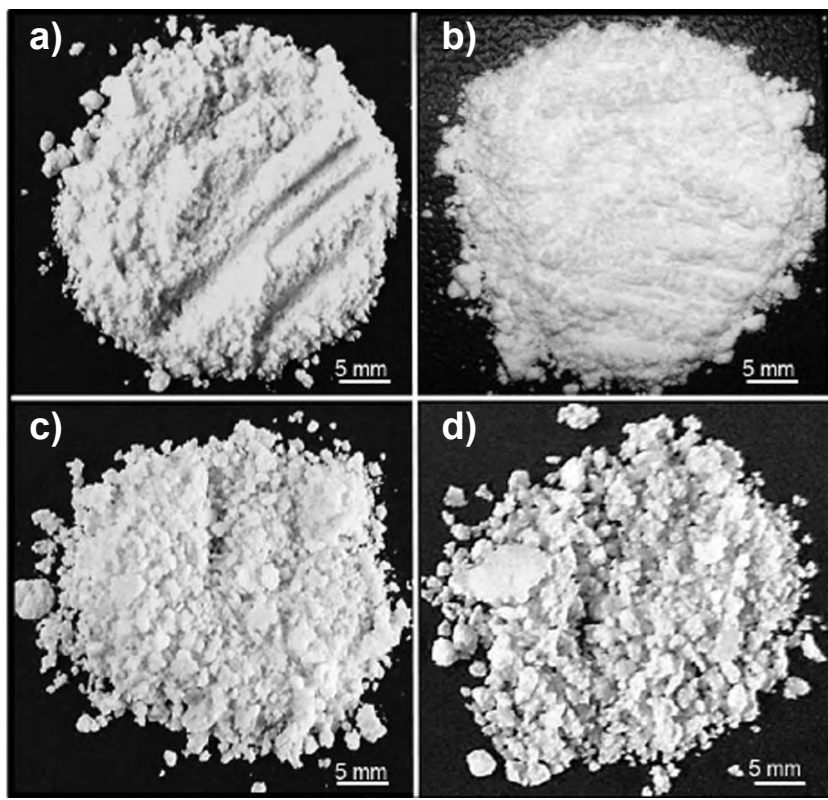

Figura 1: Fotografias digitais mostrando a morfologia macroscópica de: a) L-CHI20/HA80; b) M-CHI20/HA80; c) L-CHI/50HA50; e d) M-CHI50/HA50.

[Figure 1: Digital photographs showing the macroscopic morphology of: a) L-CHI2O/HA80; b) M-CHI20/HA80; c) L-CHI/5OHA5O; and d) M-CHI5O/HA50.] observar e classificar algumas bandas relativas às vibrações características dos grupos funcionais presentes na estrutura dos biomateriais. Os espectros FTIR das amostras da HA, L-CHI, M-CHI e dos compósitos L-CHI50 e M-CHI50 estão representadas na Fig. 3 (L-CHI20 e M-CHI20 não estão representadas porque apresentaram o mesmo espectro das amostras exibidas). O espectro infravermelho de HA (Fig. $3 \mathrm{~A}$ ) exibiu as bandas de absorção típicas na faixa de $3568 \mathrm{~cm}^{-1}$ (estiramento de $\mathrm{OH}$ ) e $631 \mathrm{~cm}^{-1}$ (vibração de $\mathrm{OH}$ ). As bandas largas em cerca de 3430 e $1645 \mathrm{~cm}^{-1}$ foram devidas à água adsorvida (alongamento e flexão de $\mathrm{HOH}$, respectivamente). Também apresentou as bandas de absorção típicas do grupo $\mathrm{PO}_{4}$ em HA a $1000-1100 \mathrm{~cm}^{-1}\left(v_{3}\right)$ e $962 \mathrm{~cm}^{-1}\left(v_{1}\right)$ [30]. As bandas de absorção em 604 e $565 \mathrm{~cm}^{-1}$ corresponderam ao modo $v_{4}$ do grupo $\mathrm{PO}_{4}$. As bandas que apareceram em 1456, 1418 e $874 \mathrm{~cm}^{-1}$ indicaram a presença de íons de carbonato de tipo B na apatita [31]. O benefício da presença de carbonato na apatita sintética é que ele contribui para aumentar a resistência mecânica e a bioatividade $[32,33]$. Os espectros no infravermelho da L-CHI (Fig. 3C) e da M-CHI (Fig. 3B) exibiram as bandas de absorção distintas em 2942-2784 $\mathrm{cm}^{-1}$ (banda de alongamento alifática $\mathrm{C}-\mathrm{H}$ ), $1657 \mathrm{~cm}^{-1}$ (amida I), $1597 \mathrm{~cm}^{-1}$ (flexão de $\mathrm{NH}_{2}$ ) e $1321 \mathrm{~cm}^{-1}$ (amida III). As bandas de absorção em $1154 \mathrm{~cm}^{-1}$ (alongamento antissimétrico da ponte C-O-C), 1082 e $1032 \mathrm{~cm}^{-1}$ (vibrações esqueléticas
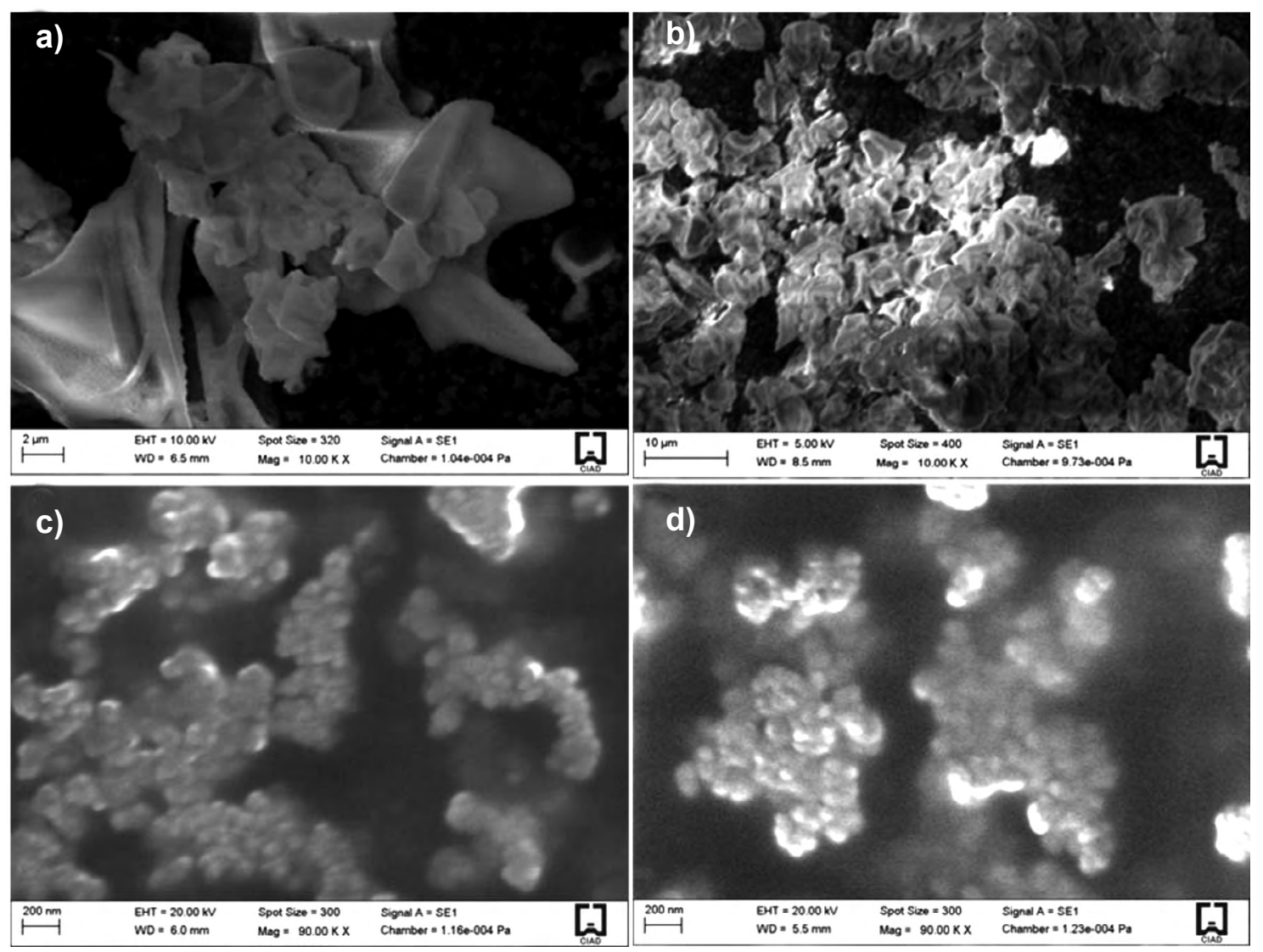

Figura 2: Micrografias eletrônicas de varredura demostrando a superfície de: a) L-CHI20/HA80; b) M-CHI20/HA80; c) L-CHI50/HA50; e d) M-CHI50/HA50.

[Figure 2: Scanning electron micrographs showing the surface of: a) L-CHI20/HA80; b) M-CHI20/HA80; c) L-CHI50/HA50; and d) M-CHI50/HA50.] 
envolvendo alongamento C-O) são características da sua estrutura sacarídea [34]. Pôde-se apreciar que os espectros de FTIR dos compósitos M-CHI50/HA50 e L-CHI50/HA50 (Figs. 3D e 3E) exibiram as bandas de absorção de ambos os componentes. Estes resultados confirmaram que não foi identificada a presença de contaminantes nas amostras e que houve formação de $\mathrm{HA}$ em associação com a CHI nos compósitos, com a presença dos grupos característicos dos materiais de origem.

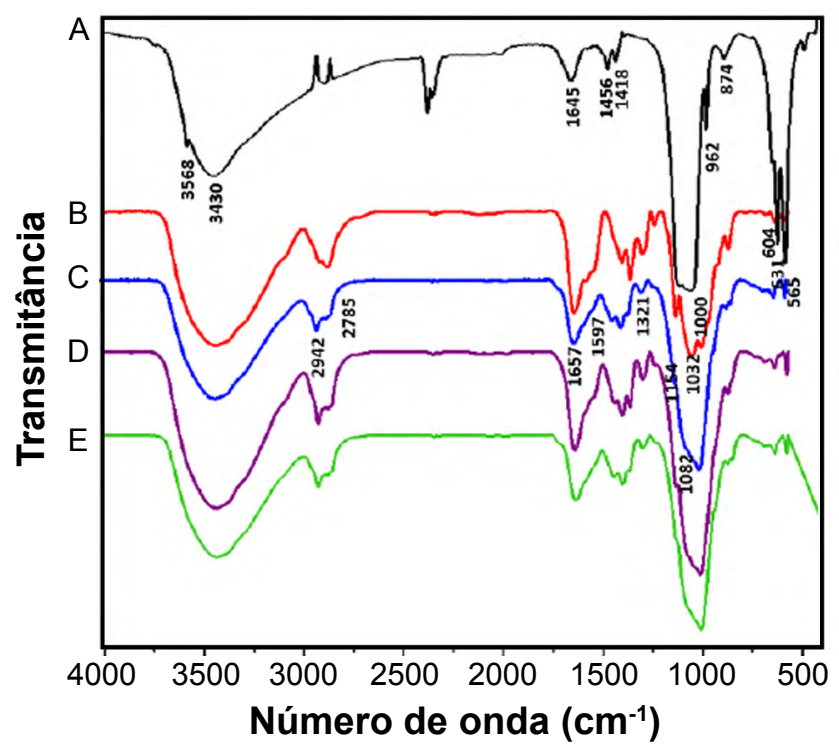

Figura 3: Espectros de FTIR das amostras de: materiais de origem HA (A), M-CHI (B) e L-CHI (C); e compósitos M-CHI50/HA50 (D) e L-CHI50/HA50 (E).

[Figure 3: FTIR spectra of samples of: starting biomaterials $H A$ (A), $\mathrm{M}$ - $\mathrm{CHI}(\mathrm{B})$, and $\mathrm{L}-\mathrm{CHI}(\mathrm{C})$; and composites $\mathrm{M}$-CHI50/HA5O (D), and L-CHI5O/HA5O (E).]

Nos difratogramas das amostras (Fig. 4) observaram-se os padrões de DRX dos biomateriais; notou-se um pico largo na região de $15^{\circ}$ e $30^{\circ}(2 \theta)$ correspondente aos principais picos de difração da CHI (JCPDS n $\left.{ }^{\circ} 39-1894\right)$. A presença da fase inorgânica foi evidenciada pelo pico largo de $30,3^{\circ}$ a $35,4^{\circ}$ em $2 \theta$ devido à contribuição dos picos de difração característicos da HA em $2 \theta=31,7^{\circ}(211), 32,2^{\circ}$ (112) e $32,8^{\circ}$ (300), juntamente com o pico em $2 \theta=26^{\circ}$ (002) conforme relatado no arquivo JCPDS $n^{\circ} 46-0905$. Vale ressaltar que a difração corresponde à parte cristalina, visto que porção amorfa não apresenta picos em DRX. Ou seja, a ausência destes picos característicos da $\mathrm{CHI}$ não significa que o polímero não esteja presente, mas sim que é pouco cristalino. Os resultados de DRX não apresentaram nenhuma alteração estrutural e comprovaram a presença das duas fases (CHI e HA) nos compósitos. A partir dos resultados de DRX obtevese o índice de cristalinidade (IC) das amostras (Tabela II), onde foi observado que todas apresentaram um valor de IC baixo, indicando baixa cristalinidade da HA [18]. O IC dos compósitos com quitosana de média massa molecular (M-CHI50/HA50 e M-CHI20/HA80) foi um pouco maior do que aqueles preparados com quitosana de baixa massa molecular ((L-CHI50/HA50 e L-CHI20/HA80), o que sugeriu que os biomateriais desenvolvidos possuíram uma característica amorfa predominante; isso pode ser devido ao fato de não terem sido sinterizados, o que pode favorecer uma maior biodegradabilidade.

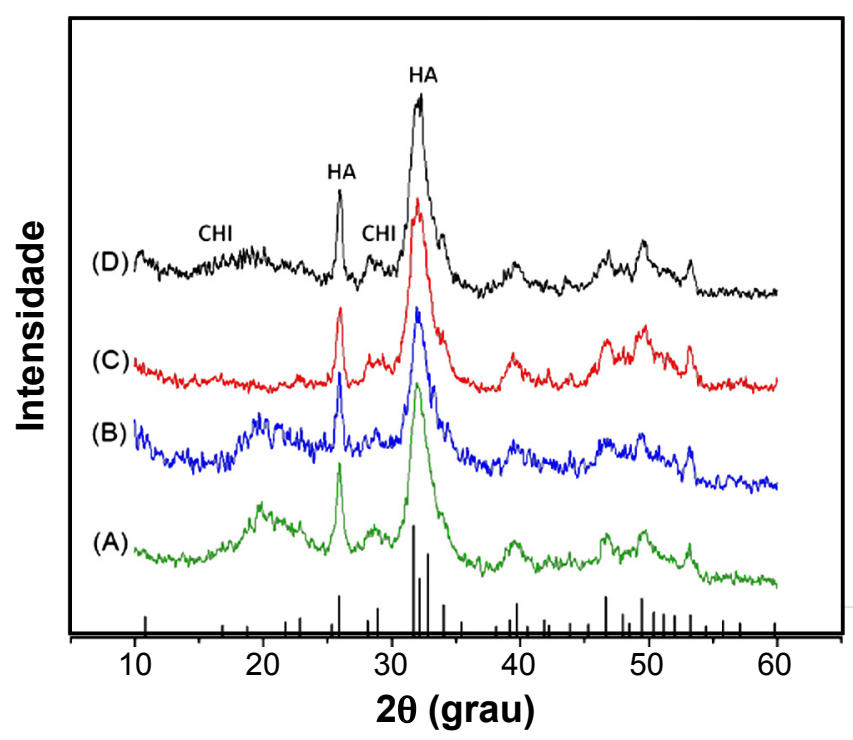

Figura 4: Padrões de difração de raios $\mathrm{X}$ dos compósitos: A) L CHI50/HA50; B) M-CHI50/HA50; C) L-CHI20/HA80; e D) M-CHI20/HA80. As linhas na parte inferior correspondem aos picos de difração da hidroxiapatita (JCPDS n ${ }^{\circ}$ 46-0905).

[Figure 4: X-ray diffraction patterns of composites: A) L-CHI5O/ HA5O; B) M-CHI5O/HA50; C) L-CHI2O/HA80; and D) M-CHI2O/ HA80. The lines at the bottom correspond to the diffraction peaks of hydroxyapatite (JCPDS No. 46-0905).]

Testes in vitro: os testes de citotoxicidade avaliam o efeito tóxico no nível celular, ou seja, a capacidade de causar morte celular, alterações na permeabilidade da membrana ou inibição enzimática. Os resultados encontrados foram importantes para avaliação das técnicas empregadas, em vista que não foi avaliada a citotoxicidade dos compósitos produzidos nos estudos anteriores em que foi empregado o método biomimético [9-11]. As percentagens de viabilidade celular $(\% \mathrm{Vc})$ deste estudo estão expressas na Fig. 5. A classificação de citotoxicidade, segundo a norma ISO 109935 [22], foi determinada de acordo com contração de $100 \%$ do extrato celular, devido o interesse do estudo em avaliar os

Tabela II - Resultados de índice de cristalinidade (IC) obtidos a partir dos padrões de DRX dos compósitos.

[Table II - Results of crystallinity index (IC) obtained from the XRD patterns of composites.]

\begin{tabular}{ccc}
\hline Amostra & $\beta_{002}$ & IC \\
\hline L-CHI50/HA50 & 0,528 & 0,094 \\
M-CHI50/HA50 & 0,449 & 0,153 \\
L-CHI20/HA80 & 0,516 & 0,101 \\
M-CHI20/HA80 & 0,441 & 0,161 \\
\hline$\beta_{002}$ - largura à meia altura da reflexão do plano (002).
\end{tabular}


biomateriais como produtos finais para utilização biomédica. Os compósitos L-CHI20/HA80 e L-CHI50/HA50 foram considerados não citotóxicos (\% Vc >90\%), e os compósitos M-CHI20/HA80 e M-CHI50/HA50 apresentaram baixa citotoxicidade (\%Vc 80-89\%). Notou-se que o tamanho de partícula e a concentração de CHI/HA nos compósitos não foram determinantes para a avaliação da citotoxicidade. $\mathrm{O}$ que resultou em diferenças entre os grupos foi a cadeia polimérica da $\mathrm{CHI}$, ocorrendo melhor viabilidade nos extratos em contato com os compósitos que possuíram CHI de baixa massa molecular (L-CHI) em sua composição. Não há um consenso na literatura sobre a massa molecular ideal para o uso da CHI como biomaterial; alguns estudos referem que a baixa massa faz com que a $\mathrm{CHI}$ ao ser hidrolisada libere unidades necessárias à biossíntese do ácido hialurônico e outros componentes da matriz extracelular, o que induz a uma tolerância tecidual e favorece o reparo [35]; outros sugerem que uma massa molecular maior contribui para ação anti-inflamatória, antitumoral e atividade imunomoduladora [36]. Estes resultados foram similares a outro estudo que ao avaliar scaffolds de HA/CHI detectaram que os arcabouços induziram a um decréscimo na morte celular quando comparados ao grupo controle, afirmando desta forma que foi possível combinar propriedades osteocondutora da HA com possíveis propriedades anti-inflamatórias da CHI [37].

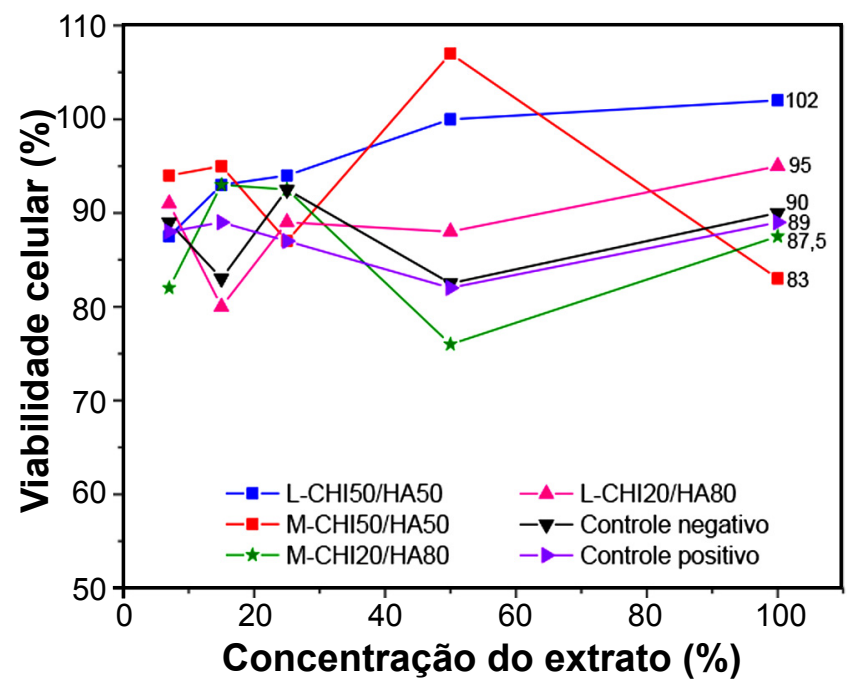

Figura 5: Percentual de viabilidade celular para determinação da citotoxicidade in vitro.

[Figure 5: Percentage of cell viability to determine in vitro cytotoxicity.]

\section{CONCLUSÕES}

As adaptações realizadas no método biomimético foram eficazes para incorporação da hidroxiapatita nanoestruturada na matriz polimérica da quitosana. As técnicas utilizadas foram eficazes para a produção dos compósitos, micropartículas por centrifugação e nanopartículas por spray drying, com tamanhos de partículas menores ao reportado na literatura. As quitosanas e os compósitos produzidos apresentaram características físico-químicas potenciais para utilização como substitutos ósseos. In vitro os compósitos que continham quitosana de baixa massa molecular (L-CHI20/HA80 e L-CHI50/HA50) não foram citotóxicos e os com média massa molecular (M-CHI20/HA80 e M-CHI50/HA50) apresentaram baixa citotoxicidade, sendo promissores para aplicações biomédicas como substitutos ósseos.

\section{AGRADECIMENTOS}

À Coordenação de Aperfeiçoamento de Pessoal de Nível Superior (CAPES)/Brasil pelo financiamento do projeto CAPES MES-CUBA ( $\left.n^{\circ} 132 / 2011\right)$. Ao Centro de Biomateriales (BIOMAT) da Universidad de La Havana/ Cuba e à Universidade de São Paulo (USP) pela colaboração.

\section{REFERÊNCIAS}

[1] M. Gutierres, M.A. Lopes, N.S. Hussain, A.T. Cabral, L. Almeida, J.D. Santos, Arq. Med. 19, 4 (2005) 153.

[2] M. Vallet-Regi, J.M. González-Calbet, Prog. Solid. State. Chem. 32, 1-2 (2004) 1.

[3] E.Y. Kawachi, C.A. Bertran, R.R. Reis, O.L. Alves, Quim. Nova 23, 4 (2000) 518.

[4] J. Fisher, A. Reddi, in "Topics in tissue engineering", N. Ashammakhi, P. Ferretti (Eds.), Un. Oulu, Helsink (2003) 1. [5] M.S. Kim, U.W. Jung, S. Kim, J.S. Lee, I.S. Lee, S.H. Choi, J. Periodontal. Implant. Sci. 43, 1 (2013) 18.

[6] C. Peniche, Y. Solís, N. Davidenko, R. García, Biotecnol. Apl. 27, 5 (2010) 202.

[7] K.R. Mohamed, H.H. Beherei, Z.M. El-Rashidy, J. Adv. Res. 5, 2 (2014) 201.

[8] J.C.V. Ribeiro, R.S. Vieira, I.M. Melo, V.M.A. Araújo, V. Lima, Sci. World. J. 2017 (2017) 1.

[9] N. Davidenko, R.G. Carrodeguas, C. Peniche, Y. Solís, R.E. Cameron, Acta Biomater. 6, 2 (2010) 466.

[10] Y. Solís, R.G. Carrodeguas, N. Davidenko, C. Peniche, Rev. CENIC Cien. Quím. 39, 1 (2008) 17.

[11] Y. Solís, N. Davidenko, R.G. Carrodeguas, J. Cruz, A. Hernandez, M. Tomas, R.E. Cameron, C. Peniche, J. Mater. Sci. 48, 2 (2013) 841.

[12] T. Wang, Q. Hu, M. Zhou, J. Xue, Y. Luo, Res. Chem. Intermed. 511, 1 (2016) 2165.

[13] H.H.K. Xu, M.D. Weir, E.F. Burguera, A.M. Fraser, Biomaterials 27, 24 (2006) 4279.

[14] M.P. Ginebra, M. Espanol, E.B. Montufar, R.A. Perez, G. Mestres, Acta Biomater. 6, 8 (2010) 2863.

[15] F.M. Klenke, Y. Liu, H. Yuan, E.B. Hunziker, K.A. Siebenrock, W. Hofstetter, J. Biomed. Mater. Res. A 85, 3 (2008) 777.

[16] M. Rinaudo, M. Milas, P. Le Dung, Int. J. Biol. Macromol. 15 (1993) 281.

[17] R.A.A. Muzzarelli, R. Rocchetti, Carbohydr. Polym. 5 (1985) 461.

[18] Y. Sa, Y. Guo, X. Feng, M. Wang, P. Li, Y. Gao, X. Yang, T. Jiang, New J. Chem. 41, 13 (2017) 5723. 
[19] B. Zhang, Y. Hou, X. Wang, Y. Wang, L. Geng, Mater. Sci. Eng. C 31, 8 (2011) 1667.

[20] K. Konopka, E. Pretzer, P.L. Felgner, N. Diizgtine, Biochim. Biophys. Acta 312 (1996) 186.

[21] ISO 10993-12, "Biological evaluation of medical devices, part 12: sample preparation and reference materials”, Int. Org. Stand., Geneva (2012).

[22] ISO 10993-5, "Biological evaluation of medical devices, part 5: tests for in vitro cytotoxicity", Int. Org. Stand., Geneva (2009).

[23] R. Signini, S.P. Campana Filho, Polímeros 8, 4 (1998) 63.

[24] M. Rinaudo, J.D. Áres, P. Le Dung, P. Thuy Binh, N.T. Dong, Carbohydr. Polym. 46 (2001) 339.

[25] A. Tolaimate, J. Desbrieres, M. Rhazi, A. Alagui, Polymer 44, 26 (2003) 7939.

[26] R.M. Fráguas, D.A. Rocha, E.R. Queiroz, C.M.P. Abreu, R.V. Sousa, E.N. Oliveira Júnior, Polímeros 25, 2 (2015) 205.

[27] A.E.C. Fai, T.C.M. Stamford, T.M. Stamford-Arnaud, P.D. Santa-Cruz, M.C.F. Silva, G.M. Campos-Takaki, T.L.M. Stamford, Molecules 16 (2011) 7143.

[28] J.E. Santos, J.P. Soares, E.R. Dockal, Polímeros 13, 4
(2003) 242.

[29] T. Forbes, M.A. García, E. Gonzalez, Ciênc. Tecnol. Aliment. 31, 4 (2011) 835.

[30] B. Fowler, Inorg. Chem. 13, 1 (1974) 194.

[31] C.E. Wilson, M.C. Kruyt, J.D. Bruijn, C.A. van Blitterswijk, F.C. Oner, A.J. Verbout, W.J.A. Dhert, Biomaterials 27 (2006) 302.

[32] J.C. Merry, I.R. Gibson, S.M. Best, W. Bonfield, J. Mater. Sci. Mater. Med. 9 (1998) 779.

[33] W.R. Walsh, D.P. Labrador, H.D. Kim, N. Guzelsu, Ann. Biomed. Eng. 22 (1994) 404.

[34] W. Arguelles-Monal, C. Peniche-Covas, Makromol. Chem. Rapid Commun. 9 (1988) 693.

[35] Y. Hidaka, M. Ito, K. Mori, H. Yagasaki, A.H. Kafrawy, J. Biomed. Mater. Res. 46, 3 (1999) 418.

[36] M.M. Mecwan, G.E. Rapalo, S.R. Mishra, W.O. Haggard, J.D. Bumgardner, J. Biomed. Mater. Res. A 97, 1 (2011) 66.

[37] V. Brun, C. Guillaume, S.M. Alami, J. Josse, J. Jing, F. Draux, S. Bouthors, D. Laurent-Maquin, S.C. Gangloff, H. Kerdjoudj, F. Velard, Bio. Med. Mater. Eng. 24 (2014) 63.

(Rec. 13/11/2018, Rev. 07/02/2019, 10/05/2019, Ac. 15/05/2019) 